\title{
Transcriptional analysis of the response of Neurospora crassa to phytosphingosine reveals links to mitochondrial function
}

\begin{abstract}
Correspondence
Arnaldo Videira

avideira@ibmc.up.pt
\end{abstract}

Received 1 April 2009

Revised 8 June 2009

Accepted 9 June 2009

\author{
Arnaldo Videira, ${ }^{1,2}$ Takao Kasuga, ${ }^{3}$ Chaoguang Tian, ${ }^{3}$ Catarina Lemos, ${ }^{1,4}$ \\ Ana Castro ${ }^{1,4}$ and N. Louise Glass $^{3}$ \\ ${ }^{1}$ IBMC - Instituto de Biologia Molecular e Celular, Universidade do Porto, Rua do Campo Alegre \\ 823, 4150-180 Porto, Portugal \\ ${ }^{2}$ ICBAS - Instituto de Ciências Biomédicas de Abel Salazar, Largo Prof. Abel Salazar 2, 4099-003 \\ Porto, Portugal \\ ${ }^{3}$ Department of Plant and Microbial Biology, University of California, Berkeley, CA 94720-3102, \\ USA \\ ${ }^{4}$ UFP - Faculdade de Ciências da Saúde, Universidade Fernando Pessoa, Rua Carlos da Maia 296, \\ 4200-150 Porto, Portugal
}

Treatment of Neurospora crassa cells with phytosphingosine (PHS) induces programmed cell death (PCD) by an unknown mechanism. To determine the relationship between PHS treatment and PCD, we determined changes in global gene expression levels in N. crassa during a time-course of PHS treatment. Most genes having differential expression levels compared to untreated samples showed an increase in relative expression level upon PHS exposure. However, genes encoding mitochondrial proteins were highly enriched among $\sim 100$ genes that showed a relative decrease in expression levels after PHS treatment, suggesting that repression of these genes might be related to the death-inducing effects of PHS. Since mutants in respiratory chain complex I are more resistant to both $\mathrm{PHS}$ and hydrogen peroxide $\left(\mathrm{H}_{2} \mathrm{O}_{2}\right)$ than the wild-type strain, possibly related to the production of reactive oxygen species, we also compared gene expression profiles of a complex I mutant (nuo14) and wild-type in response to $\mathrm{H}_{2} \mathrm{O}_{2}$. Genes with higher expression levels in the mutant, in the presence of $\mathrm{H}_{2} \mathrm{O}_{2}$, are also significantly enriched in genes encoding mitochondrial proteins. These data suggest that complex I mutants cope better with drug-induced decrease in expression of genes encoding mitochondrial proteins and may explain their increased resistance to both $\mathrm{PHS}$ and $\mathrm{H}_{2} \mathrm{O}_{2}$. As a way of identifying new components required for PHS-induced death, we analysed the PHS sensitivity of 24 strains carrying deletions in genes that showed a significant alteration in expression pattern when the wild-type was exposed to the sphingolipid. Two additional mutants showing increased resistance to PHS were identified and both encode predicted mitochondrial proteins, further supporting the role of the mitochondria in PHS-induced PCD.

\section{INTRODUCTION}

In response to endogenous or external stimuli, living cells can undergo a genetic programme eventually leading to death, commonly known as programmed cell death (PCD). This suicide process can take several forms (apoptosis,

Abbreviations: PCD, programmed cell death; PHS, phytosphingosine; $\mathrm{ROS}$, reactive oxygen species.

The microarray data associated with this paper have been deposited at the Yale Microarray Experimental Design site (http://www.yale.edu/ townsend/Links/ffdatabase/introduction.html) under Experiment ID 48.

A supplementary table showing mRNA profiling results and functional annotations is available with the online version of this paper. necrosis, autophagy), each of which displays typical characteristics at both the cellular and molecular level. These processes can be interchangeable and are sometimes difficult to distinguish. In metazoan organisms, the death programme is essential for development and its dysfunction may result in human disease, such as cancer. In addition to multicellular eukaryotic species, both prokaryotic and eukaryotic unicellular organisms also undergo PCD (Cheng et al., 2008; Green \& Kroemer, 2004; Hamann et al., 2008; Madeo et al., 2004).

Mitochondria are cellular organelles, still possessing their own genome, that are responsible for the production of most cellular energy in eukaryotes. This production occurs 
mainly in the mitochondrial inner membrane through the process of oxidative phosphorylation, which involves the components of the respiratory chain and ATP synthase (Hatefi, 1985). The involvement of mitochondria and specific mitochondrial proteins, particularly components of the respiratory chain, in most cases of PCD has been well established. This includes, for example, the generation of reactive oxygen species (ROS) and the release of mitochondrial proteins (Eisenberg et al., 2007; Pereira et al., 2008; Skulachev, 2006).

Many of the proteins and mechanisms related to PCD have been intensively studied and characterized. Much less explored has been the analysis of gene expression during the death process (Fedorova et al., 2005; Laun et al., 2005). However, it is becoming increasingly clear that PCD may involve complex metabolic networks and is a more complicated cellular process than initially thought (Kimchi, 2007). These observations imply that many connected gene products remain to be identified. In addition, it is likely that different organisms, especially eukaryotic microbes, may vary in both the mechanism and the suite of responses associated with PCD. Although work in Saccharomyces cerevisiae has identified a number of fungal homologues of mammalian 'PCD' genes (Eisenberg et al., 2007; Madeo et al., 2004), filamentous fungi contain additional PCD genes (Fedorova et al., 2005). Filamentous fungi have more similarities to higher eukaryotes (e.g. multicellularity) and thus represent an excellent alternative system to investigate the process. The discovery of novel proteins involved in PCD and their targeting, with, for example, inhibitors, will be very useful for the modulation of cell killing.

We are interested in the overall analysis of gene expression during drug-induced PCD as a means of finding novel proteins and metabolic pathways involved in the process. We use the filamentous fungus Neurospora crassa as a model organism because it offers many experimental advantages (Davis \& Perkins, 2002; Dunlap et al., 2007) and PCD can be induced by different means (Castro et al., 2008; Glass \& Dementhon, 2006; Lu, 2006). Moreover, the ongoing project for the disruption of all predicted genes in the N. crassa genome (Colot et al., 2006) allows direct testing of the sensitivity of specific mutants to PCDinducing stimuli, including mutants in genes identified by expression profiling. In filamentous fungi, the sphingolipid phytosphingosine (PHS) induces PCD with the involvement of mitochondria (Castro et al., 2008; Cheng et al., 2003; Woodcock, 2006). In this article, we present our analysis of the transcriptional response to the deathinducer PHS in $N$. crassa and its major effect on genes encoding mitochondrial proteins. We further show that this type of analysis is useful to identify novel components associated with PCD in filamentous fungi.

\section{METHODS}

Growth conditions and experimental design. The wild-type $N$. crassa sequenced strain FGSC2489 (Galagan et al., 2003) and the gene deletion strains generated by the Neurospora Genome Project (Colot et al., 2006) were obtained from the Fungal Genetics Stock Center (FGSC) (McCluskey, 2003). The complex I mutant nuol4 (Marques et al., 2005), the AMID ( $\triangle N C U 06061$; FGSC12090) deletion strain (Castro et al., 2008) and the general handling of the fungal strains (Davis \& de Serres, 1970) have been described before. For the determination of drug sensitivity in spot assays, conidia from different strains were harvested with distilled water and adjusted to a concentration of $6.56 \times 10^{7}$ cells $\mathrm{ml}^{-1}$. Threefold serial dilutions of each conidial suspension were then spotted on the appropriate GFS plates (Davis \& de Serres, 1970) and incubated at $26{ }^{\circ} \mathrm{C}$, as detailed before (Castro et al., 2008).

Conidia for microarrays were obtained from cultures grown for 7 days in Vogel's minimal medium (Davis \& de Serres, 1970) at $25^{\circ} \mathrm{C}$ under constant light (Kasuga et al., 2005). To study the effects of PHS on gene expression, conidia $\left(10^{7} \mathrm{ml}^{-1}\right)$ were germinated for $5 \mathrm{~h}$ in Vogel's minimal medium at $30{ }^{\circ} \mathrm{C}$ with strong agitation. Subsequently, PHS was added $\left(10 \mu \mathrm{g} \mathrm{ml}^{-1}\right)$ from a stock solution in ethanol $\left(4 \mathrm{mg} \mathrm{ml}^{-1}\right)$ and the incubation continued for different times. The mycelium was collected by quick filtration, frozen in liquid nitrogen and kept at $-70{ }^{\circ} \mathrm{C}$.

For assessing the effect of hydrogen peroxide $\left(\mathrm{H}_{2} \mathrm{O}_{2}\right)$ treatment on gene expression profiles, strains were inoculated onto large square Petri dishes that were halved with a barrier. One side was filled with Bird's medium (Metzenberg, 2004), while the other side of the Petri dish contained either Bird's medium or Bird's medium with $4 \mathrm{mM}$ $\mathrm{H}_{2} \mathrm{O}_{2}$. The large Petri dishes were then covered with a cellophane sheet. Strains were inoculated onto the first half of the Petri dish and allowed to grow $1 \mathrm{~cm}$ into the half of the Petri dish containing no treatment (Bird's medium) or treatment (Bird's medium plus $4 \mathrm{mM}$ $\mathrm{H}_{2} \mathrm{O}_{2}$ ). A $1 \mathrm{~cm}$ wide cellophane strip containing hyphae at the periphery of the colony was sliced from each plate (treatment and no treatment), frozen in liquid nitrogen and kept at $-70{ }^{\circ} \mathrm{C}$.

Closed-circuit designs were employed for microarray comparisons (Fig. 1), because they are statistically robust and provide a higher

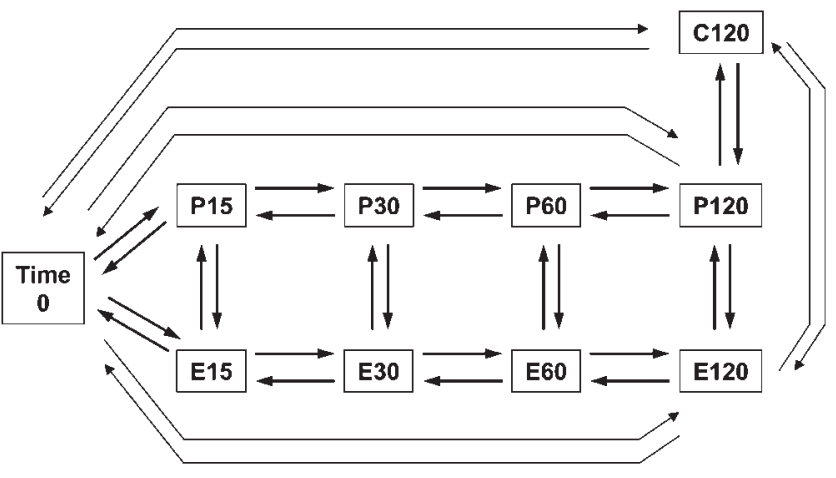

Fig. 1. Experimental design for a time-course assessing gene expression patterns of germinating $N$. crassa conidia exposed to PHS. Conidia were germinated in minimal medium for $5 \mathrm{~h}$ (time 0) and then incubated in the absence $(C)$ or in the presence of $10 \mu \mathrm{g}$ PHS $\mathrm{ml}^{-1}$ in $0.25 \%$ ethanol (P) or ethanol alone (E). Samples were withdrawn at the indicated time points (minutes) and used for microarray hybridization and quantification of gene expression levels. The arrows indicate the di-swap hybridizations, with the arrowhead pointing to the sample labelled with Cy5 (details are described in Methods). 
resolution in identifying differentially expressed genes than designs that use a universal reference (Townsend, 2003).

RNA and cDNA processing. RNA was isolated from the fungal mycelia with TRIzol (Invitrogen Life Technologies), purified with the RNeasy kit (Qiagen) and used for the synthesis of cDNA with the Pronto kit (Corning), according to the manufacturers' instructions. The cDNA was labelled with either $\mathrm{Cy} 3$ or Cy5 dye (Amersham), dried under vacuum, resuspended in hybridization solution, heated at $95{ }^{\circ} \mathrm{C}$ for $5 \mathrm{~min}$ and used for hybridization. Each hybridization was duplicated, labelling one cDNA sample with $\mathrm{Cy} 3$ and the other with Cy5, and vice versa (di-swap).

Microarray slide hybridization. We used $\gamma$-aminopropylsilane slides printed with 70-mer oligonucleotides, which include the 10526 ORFs predicted in the Neurospora genome (Kasuga et al., 2005; Kasuga \& Glass, 2008; Tian et al., 2007). The Pronto kit (Corning) was used for hybridization, following the instruction procedures included. Briefly, after presoak and prehybridization steps of the slides to reduce background, the labelled cDNA was laid on the slides covered with a LifterSlip cover glass (Erie Scientific). Hybridization was performed for $16 \mathrm{~h}$ at $42{ }^{\circ} \mathrm{C}$, followed by washing steps to remove unbound DNA.

Microarray data acquisition and analysis. The hybridization images were obtained with a GenePix 4000B scanner and the signals were quantified with the GenePix Pro6 software, which automatically flagged low-quality spots. Then, slides were also inspected manually. Spots with a mean fluorescence intensity for at least one of the Cy3 or Cy5 dyes that was greater than the mean background intensity plus three standard deviations were selected for further analysis if less than $0.02 \%$ of the pixels were saturated. Normalized ratio data were analysed with the Bayesian Analysis of Gene Expression Levels (BAGEL) software in order to calculate a relative expression level and a credible interval for each gene in each sample (Townsend \& Hartl, 2002). The profiles of gene expression were clustered with Hierarchical Clustering Explorer 3.0 (Frishman et al., 2001; Seo \& Shneiderman, 2002), using Pearson's correlation coefficient to measure similarities in expression patterns between genes. Consequently, the closest two genes or clusters became successively joined. The genes were associated with functional categories using the FunCat catalogue created by MIPS (Frishman et al., 2001; Ruepp et al., 2004). The statistically significant enrichment of gene groups in these categories relative to all genes with detected mRNA profiles was determined by Fisher's exact test in the statistical software R 2.6 (http://bioconductor.org), as detailed by Kasuga \& Glass (2008). A significance level of 0.05 was used with multiple testing corrections according to Benjamini \& Hochberg (1995). Microarray data have been deposited at the Yale Microarray Experimental Design site (http://www.yale. edu/townsend/Links/ffdatabase/introduction.html) under Experiment ID 48. Supplementary Table S1 (available with the online version of this paper) lists mRNA profiling results and functional annotations.

\section{RESULTS}

\section{Gene expression in the presence of PHS}

PHS induces rapid PCD in N. crassa (Castro et al., 2008). In order to analyse the transcriptional response to this drug, we set up the time-course experiment depicted in Fig. 1. Conidia were inoculated into liquid medium and, after a germination period of $5 \mathrm{~h}$ (Kasuga et al., 2005), were further incubated in the presence or absence of PHS for $2 \mathrm{~h}$. Samples were collected at different time points during the $2 \mathrm{~h}$ time-course and used to prepare fungal RNA for evaluation of relative expression levels using fullgenome N. crassa microarrays (Kasuga et al., 2005; Kasuga \& Glass, 2008; Tian et al., 2007). In these experiments, we obtained expression data for 6011 genes, representing more than half of the fungal genome (Supplementary Table S1, page 1). In a parallel experiment, we assessed the effects of PHS on cell death by methylene blue staining (Fleissner \& Glass, 2007). Nuclei from live cells are not stained by the dye. Upon exposure to PHS, cell death is induced in a time-dependent manner (Fig. 2).

The time-course profiles of gene expression were individually inspected and those genes displaying differential expression in the presence or absence of PHS were selected for further analysis. At least 762 genes $(\sim 13 \%)$ were influenced by PHS treatment, indicating that an active transcriptional response to drug exposure occurs (Supplementary Table S1, page 2). This set of affected genes was grouped by clustering analysis and the results are shown in Fig. 3. Three typical expression profiles were observed: genes that showed an early increase in relative expression level following exposure to PHS (15-30 min treatment, 272 genes), genes whose relative expression levels increased later following exposure to PHS (60$120 \mathrm{~min}, 387$ genes) and genes that showed a relative decrease in expression level following exposure to PHS (103 genes).

The 762 genes that showed differential relative expression levels when $N$. crassa cells were exposed to PHS were assigned to a functional category, as defined by the FunCat catalogue (Ruepp et al., 2004). We evaluated whether specific functional categories were statistically over-represented in each of the 'early-induced', 'late-induced' and 'repressed' gene categories (Table 1). As could be anticipated as a death-inducer effect, an enrichment for

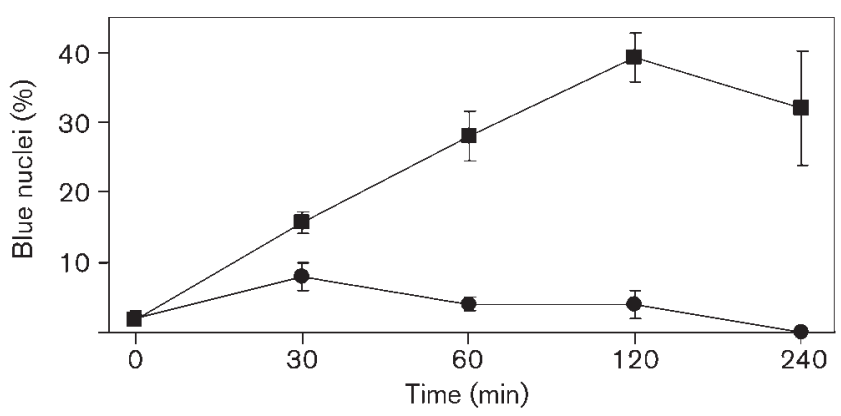

Fig. 2. Analysis of cell death induced by PHS in $N$. crassa. Conidia were germinated in minimal medium for $3.5 \mathrm{~h}$ (time 0 ) and then incubated in the presence of $10 \mu \mathrm{g} \mathrm{PHS} \mathrm{ml} \mathrm{ml}^{-1}$ in $0.25 \%$ ethanol ( $\boldsymbol{\square}$ ) or ethanol alone ( $)$. Samples were withdrawn at the indicated time points and the percentage of cell nuclei that stained with $0.003 \%(\mathrm{w} / \mathrm{v})$ methylene blue (a vital dye) was determined. Standard deviations from three experiments are shown. 


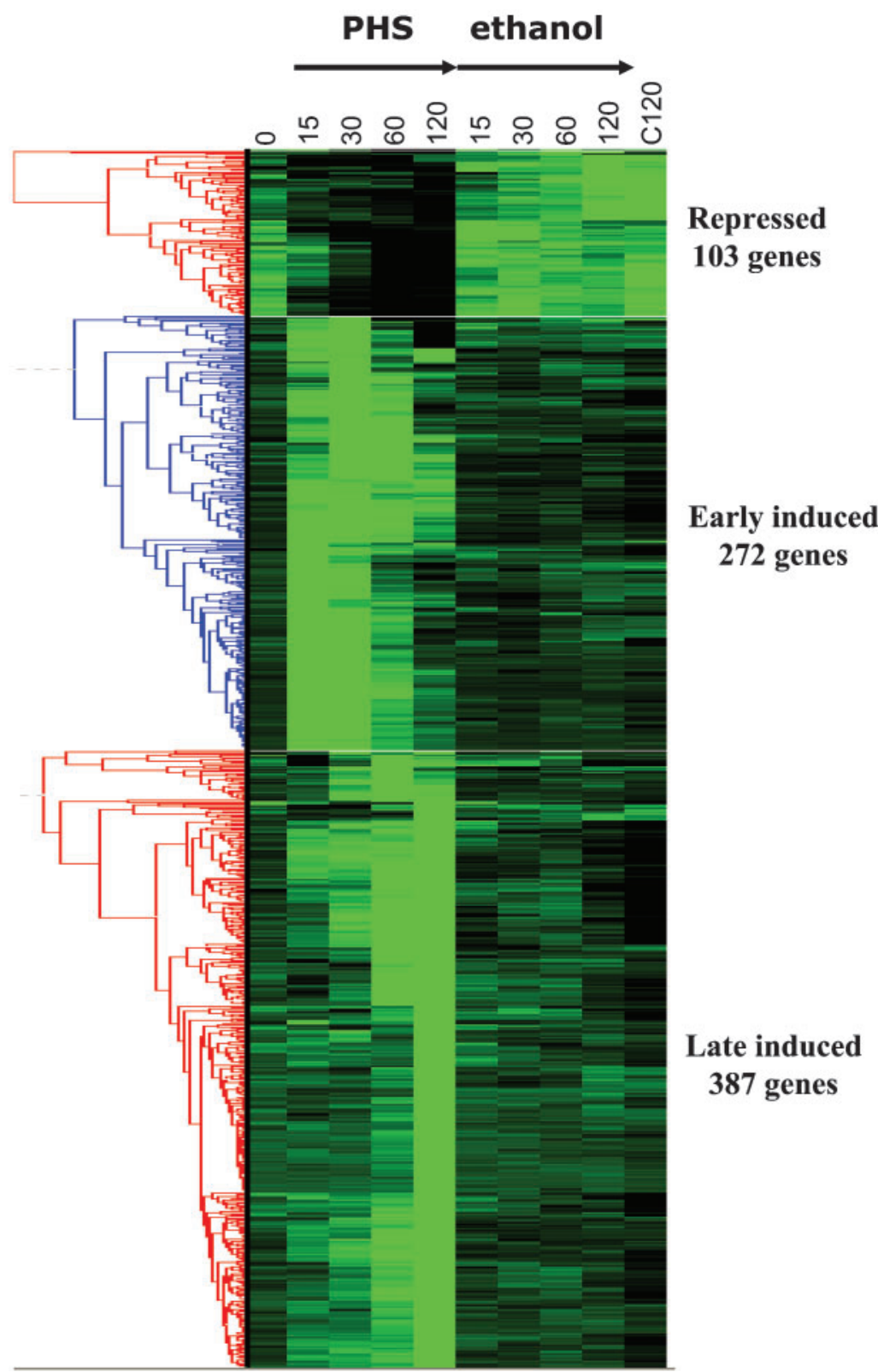

Fig. 3. Profile of the time-course of differential gene expression patterns in N. crassa germinating conidia upon PHS treatment. The quantification of expression levels was obtained from experiments as described in Fig. 1. Genes that showed differential expression across the time-course in the presence or absence of PHS were clustered according to their similarity of expression patterns. The green and black colours indicate high and low expression levels, respectively.

genes induced early following treatment with PHS are predicted to be involved in cell rescue and stress response, protein degradation and modification, and the glyoxylate cycle, involved in lipid metabolism. Functional categories that included predicted cell cycle and DNA processing proteins, signal transduction and protein fate were enriched among genes that showed a later increase in relative expression level when $N$. crassa was exposed to PHS. However, the most dramatic effect of treatment of $N$. crassa with PHS appears to be a decrease in the expression level of genes encoding mitochondrial proteins, in particular those involved in oxidative phosphorylation. Both nuclear and cytoplasmic genes coding for mitochondrial proteins were highly enriched in the gene set repressed by PHS treatment (Supplementary Table S1, page 3). The statistical significance of this enrichment can be inferred from the extremely low P-values (Table 1).

\section{Comparison of the wild-type and nuo14 response to $\mathrm{H}_{2} \mathrm{O}_{2}$}

We previously determined that strains containing mutations in complex I were more resistant to treatment with PHS or $\mathrm{H}_{2} \mathrm{O}_{2}$, although strains containing mutations in other components of the respiratory chain did not show a similar resistance to either treatment (Castro et al., 2008). These observations suggest that the resistance of the complex I mutants to PHS and to $\mathrm{H}_{2} \mathrm{O}_{2}$ include similar mechanisms. We therefore investigated whether this resistance was associated with differential gene expression. 
Table 1. Functional categories over-represented among genes displaying differential expression upon PHS treatment

\begin{tabular}{|c|c|c|c|c|}
\hline \multirow[t]{2}{*}{ Description } & \multicolumn{2}{|c|}{ No. of genes in the cluster } & \multirow{2}{*}{$\begin{array}{l}\text { Total no. of } \\
\text { genes }\end{array}$} & \multirow[t]{2}{*}{$P$-value ${ }^{*}$} \\
\hline & Obs $\dagger$ & $\operatorname{Exp} \dagger$ & & \\
\hline \multicolumn{5}{|l|}{ Early induced (272) } \\
\hline 02.04 Glyoxylate cycle & 3 & 0.3 & 6 & $3.97 \mathrm{E}-02$ \\
\hline 14 PROTEIN FATE (folding, modification, destination) & 36 & 21 & 454 & $2.90 \mathrm{E}-02$ \\
\hline 14.13 Protein/peptide degradation & 16 & 5 & 109 & $1.33 \mathrm{E}-03$ \\
\hline 14.13.01 Cytoplasmic and nuclear protein degradation & 7 & 1.7 & 36 & $2.93 \mathrm{E}-02$ \\
\hline 14.13.01.01 Proteasomal degradation (ubiquitin/proteasomal pathway) & 5 & 0.9 & 19 & $3.35 \mathrm{E}-02$ \\
\hline 32 CELL RESCUE, DEFENCE AND VIRULENCE & 20 & 8.8 & 191 & $1.82 \mathrm{E}-02$ \\
\hline 32.01 Stress response & 14 & 4 & 86 & $1.33 \mathrm{E}-03$ \\
\hline \multicolumn{5}{|l|}{ Late induced (387) } \\
\hline 10 CELL CYCLE AND DNA PROCESSING & 30 & 16.6 & 252 & $3.59 \mathrm{E}-02$ \\
\hline 10.03 Cell cycle & 21 & 10.3 & 156 & $3.52 \mathrm{E}-02$ \\
\hline 14 PROTEIN FATE (folding, modification, destination) & 54 & 29.9 & 454 & $5.12 \mathrm{E}-04$ \\
\hline 30.05 Transmembrane signal transduction & 4 & 0.6 & 9 & $3.84 \mathrm{E}-02$ \\
\hline 70.10 Nucleus & 29 & 15.4 & 234 & $2.96 \mathrm{E}-02$ \\
\hline \multicolumn{5}{|l|}{ Repressed (103) } \\
\hline 02 ENERGY & 48 & 2.8 & 162 & $1.44 \mathrm{E}-46$ \\
\hline 02.07 Pentose-phosphate pathway & 3 & 0.1 & 8 & $9.26 \mathrm{E}-03$ \\
\hline 02.11 Electron transport and membrane-associated energy conservation & 29 & 0.9 & 49 & $1.14 \mathrm{E}-37$ \\
\hline 02.13 Respiration & 35 & 1.2 & 67 & $2.38 \mathrm{E}-43$ \\
\hline 02.13.03 Aerobic respiration & 16 & 0.4 & 25 & $7.04 \mathrm{E}-21$ \\
\hline 02.45 Energy conversion and regeneration & 3 & 0.2 & 13 & $3.25 \mathrm{E}-02$ \\
\hline 02.45.15 Energy generation (e.g. ATP synthase) & 3 & 0.2 & 10 & $1.87 \mathrm{E}-02$ \\
\hline 20.01 Transported compounds (substrates) & 24 & 4.1 & 234 & $7.07 \mathrm{E}-11$ \\
\hline 20.01.15 Electron transport & 19 & 0.7 & 39 & $7.74 \mathrm{E}-22$ \\
\hline 20.03 Transport facilities & 16 & 2.3 & 131 & $4.00 \mathrm{E}-08$ \\
\hline 70.16 Mitochondrion & 44 & 4.8 & 274 & $4.98 \mathrm{E}-30$ \\
\hline
\end{tabular}

${ }^{\star} P$-values according to Fisher's exact test with Benjamini \& Hochberg (1995) multiple testing correction.

$\dagger$ Obs, observed; Exp, expected. 'Exp' is the number of genes predicted to be in a functional category by chance from the total number of genes whose expression profiles were detected versus the expression profile from the number of genes actually observed ('Obs').

We compared the response of wild-type and a complex I mutant (nuo14) to treatment with $\mathrm{H}_{2} \mathrm{O}_{2}$; the resistance of complex I mutants towards $\mathrm{H}_{2} \mathrm{O}_{2}$ is more marked than their resistance towards PHS (Castro et al., 2008). Our experimental design is illustrated in Fig. 4(A). In these experiments, we obtained microarray signal data for 5709 genes (Supplementary Table S1, page 4).

Although the $\mathrm{H}_{2} \mathrm{O}_{2}$ concentration used $(4 \mathrm{mM})$ was lethal to conidia (data not shown), the morphological response of $N$. crassa mycelia to $\mathrm{H}_{2} \mathrm{O}_{2}$ treatment was relatively mild. The expression of catalase- 3 is elevated 1.5-2-fold in response to $\mathrm{H}_{2} \mathrm{O}_{2}$ (Fig. 4B). This protein is predicted to be exported from cells and is thus likely to be involved in the removal of the $\mathrm{H}_{2} \mathrm{O}_{2}$ present in the culture medium. In the gene set from either wild-type or nuo14 that showed altered expression profiles in response to $\mathrm{H}_{2} \mathrm{O}_{2}$, we did not observe significant changes in the expression level of other detoxifying enzymes, such as other catalases, peroxidases, mitochondrial and cytosolic superoxide dismutases, thioredoxins or enzymes acting on glutathione. Unexpectedly, the mRNA levels of mitochondrial alternative oxidase
(NCU07953.2) and cytochrome $c$ (NCU01808.2) were 22.5 -fold higher in the complex I mutant grown in minimal medium as compared to wild-type, reminiscent of the situation in complex IV (Bertrand \& Pittenger, 1972; Descheneau et al., 2005) and complex III mutants (Duarte \& Videira, 2009). Induction of alternative oxidase is expected in complex IV and complex III mutants, but not in complex I mutants, because it represents an alternative pathway of electron flow that branches off the standard electron-transport chain after complex I, but before complexes III and IV. These results suggest that induction of alternative oxidase and cytochrome $c$ represent a general response to deficiencies in the mitochondrial respiratory chain.

Perhaps the most interesting result from the data analysis is depicted in Fig. 4(C). We considered the gene set that showed a statistically significant $(P<0.05)$ increased expression level in the complex I mutant as compared to wild-type in response to $\mathrm{H}_{2} \mathrm{O}_{2}$. The analysis of the distribution of these genes by functional category revealed that genes encoding mitochondrial proteins were the most 
A

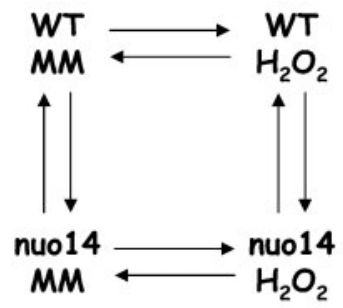

B

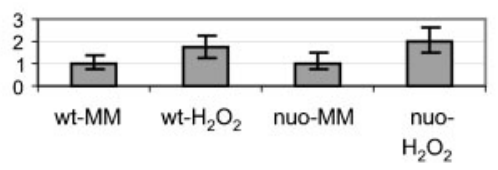

C

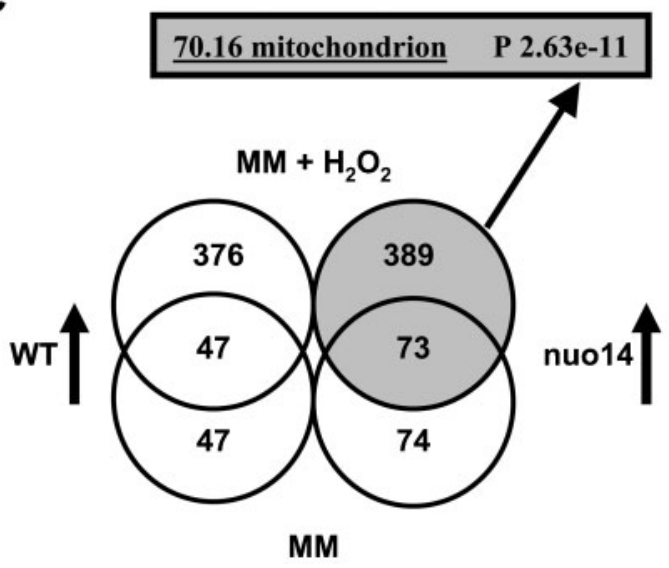

Fig. 4. Comparison of gene expression between wild-type and a complex I mutant in the presence or absence of $\mathrm{H}_{2} \mathrm{O}_{2}$. (A) Scheme of the microarray hybridizations. A mycelial strip from the wild-type strain and the nuo14 mutant that had grown into solid minimal medium (MM) or into minimal medium containing $4 \mathrm{mM} \mathrm{H}_{2} \mathrm{O}_{2}$ was used to compare gene expression levels. (B) Relative expression levels of catalase-3 (NCU00355.2). (C) Venn diagram showing the number of genes with statistically significant higher expression $(\uparrow)$ in either wild-type or the nuo14 mutant in each of the different growth conditions. A functional category with statistically significant over-representation in a group of genes in the nuo14 mutant exposed to $\mathrm{H}_{2} \mathrm{O}_{2}$ is indicated.

over-represented category within the group. These results suggest that complex I mutants may cope better than wildtype with the induced repression of genes encoding mitochondrial proteins upon exposure to PHS (as described above for the PHS effects on wild-type), and this may explain why they are more resistant to both PHS and $\mathrm{H}_{2} \mathrm{O}_{2}$.

\section{Sensitivity of selected deletion mutants to PHS}

The expression of many N. crassa genes is affected by PHS exposure, as described above. The cellular processes in which these genes participate might therefore be relevant for PHS-induced PCD. Consequently, mutant strains carrying deletions in relevant genes might respond differently to PHS than the wild-type strain. To verify this, we took advantage of an ongoing project to disrupt all N. crassa genes (Colot et al., 2006; Dunlap et al., 2007) and tested the sensitivity to PHS of several available deletion mutants in spot assays. Serial dilutions of conidia from the different strains were exposed to media containing PHS (Fig. 5). In these experiments, the complex I mutant nuo14 and a mutant lacking an AMID-like protein $(\triangle N C U 06061.2)$ were used as controls. The nuo14 mutant is more resistant to PHS, while $\triangle N C U 06061.2$ is more sensitive to PHS than the wild-type strain (Castro et al., 2008). Among the 24 mutants carrying deletions in genes whose expression is affected by PHS, we identified two strains that were more resistant to the drug than the wildtype strain (Fig. 5). The NCU00502.2 and NCU03415.2 genes encode subunit 4 of mitochondrial ATP synthase and a probable aldehyde dehydrogenase, respectively. Our results indicate their involvement in PHS-induced PCD and point to directions for future work.

\section{DISCUSSION}

We are interested in characterizing PCD mechanisms in $N$. crassa and in identifying unknown pathways involved in the process. In this work, we performed a detailed timecourse analysis of gene expression in the wild-type following exposure to the death-inducing drug PHS. Most genes displaying differential expression upon the drug treatment increased in expression level, showing that the response to PHS treatment induces a transcriptionally active process. Among this set of genes, we found enrichment for genes encoding proteins belonging to functional categories that have been described to participate in stress and death responses (Bonawitz et al., 2006; Fedorova et al., 2005; Fröhlich et al., 2007; Hamann et al., 2008; Laun et al., 2005; Lu, 2006; Lucau-Danila et al., 2005). For instance, genes encoding proteins predicted to be involved in cell rescue and stress response, cell cycle and DNA processing, protein fate and modulation and for those involved in protein degradation were significantly enriched. However, the most striking effect of PHS appears to be the repression of genes encoding mitochondrial proteins, particularly those associated with energy conversion. Both nuclear and mitochondrial genes encoding the organelle proteins were extremely enriched among the $\sim 100$ genes that showed a decrease in relative expression following PHS exposure. This is in line with the findings that mitochondria have a central role in PHS-induced PCD (Castro et al., 2008; Cheng et al., 2003; Woodcock, 2006).

Altered transcription during PCD has been noticed before. Nuclear genes encoding mitochondrial proteins represented the largest group found to be differentially regulated in apoptotic yeast (Laun et al., 2005). A reduction of yeast lifespan is associated with deficiencies in mitochondrial gene expression, which result in ROS-mediated inhibition of respiration (Bonawitz et al., 2006). A decrease in 


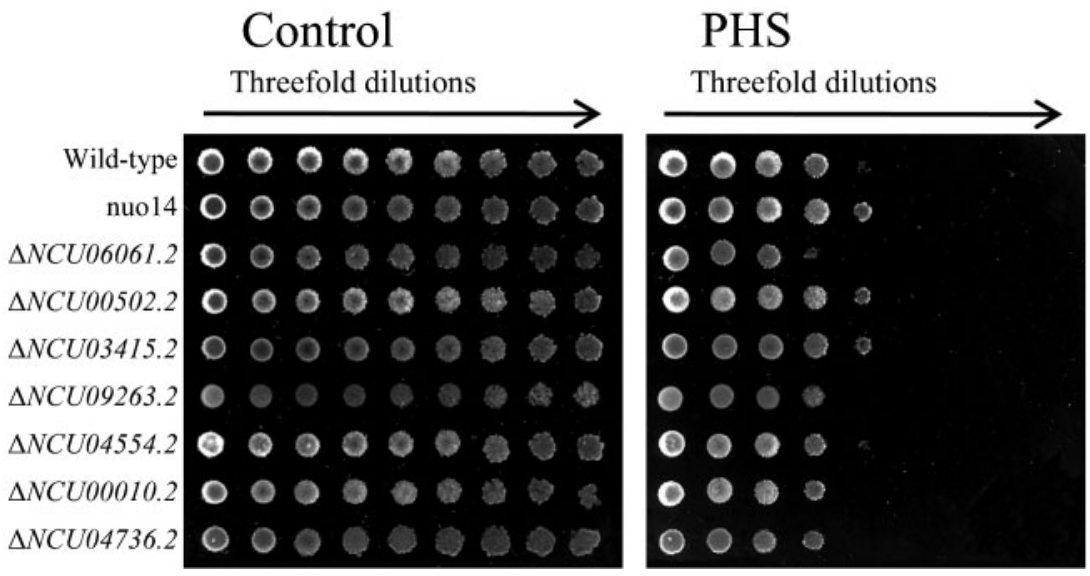

Fig. 5. Sensitivity of selected deletion strains to PHS. Serial threefold dilutions of conidia from the indicated strains were spotted on GFS medium containing $7.5 \mu \mathrm{g} \mathrm{PHS} \mathrm{ml} \mathrm{m}^{-1}$ and incubated at $25^{\circ} \mathrm{C}$. The less concentrated spots contain $\sim 50$ conidia. The sensitivity to PHS of other strains tested ( $\triangle$ NCU01376.2, $\triangle N C U 01640.2, \Delta N C U 00355.2, \Delta N C U 098-$ 82.2, $\triangle N C U 04421.2, \quad \triangle N C U 07121.2$, $\triangle N C U 06953.2, \Delta N C U 03241.2, \Delta N C U 047-$ 37.2, $\Delta$ NCU06664.2, $\Delta$ NCU01754.2, $\triangle N C U 06660.2, \Delta N C U 01645.2, \Delta N C U 004-$ 61.2, $\quad \Delta N C U 05627.2, \quad \Delta N C U 04635.2$, $\Delta$ NCU00865.2, $\triangle$ NCU03888.2) was similar to that of the wild-type (not shown).

mitochondrial gene transcripts has also been observed in patients suffering complex I deficiency (van der Westhuizen et al., 2003) and alterations in mitochondrial components were identified in a mouse model of Friedreich ataxia (Coppola et al., 2006). Here we describe, to our knowledge for the first time, a generalized downregulation of genes coding for mitochondrial proteins in response to treatment with PHS. This treatment likely creates an imbalance of mitochondrial components, eventually leading to mitochondrial dysfunction and triggering PCD. It is possible that the signalling for this regulation is mediated by ROS produced by the respiratory chain following drug exposure. First, ROS are known to regulate gene expression (LucauDanila et al., 2005) and are elevated in most cases of PCD (Robson, 2006; Skulachev, 2006). Second, we found that mutants in respiratory chain complex I are more resistant to PHS-induced death than the wild-type strain. In a previous study, resistance was correlated with a lower amount of ROS production in complex I mutants in the presence of the sphingolipid PHS (Castro et al., 2008). In the present study, we further compared gene expression patterns between a complex I mutant and wild-type Neurospora, including their responses to $\mathrm{H}_{2} \mathrm{O}_{2}$, which mimics elevated ROS generation (Bonawitz et al., 2006). We found that genes encoding mitochondrial proteins were the most enriched category among genes that showed increased expression levels in the complex I mutant as compared to wild-type in the presence of $\mathrm{H}_{2} \mathrm{O}_{2}$. This may be the reason why complex I mutants are more resistant than wild-type to PHS treatment and it supports our proposition that repression of genes encoding mitochondrial proteins is a crucial event of PHS-induced PCD. The complex I mutants are less affected in the expression of mitochondrial proteins and produce lower amounts of ROS; they may therefore be less prone to undergo PCD.

Our hypothesis was that new genes involved in PHSinduced death would be identified by transcriptional profiling. We therefore tested the PHS sensitivity of available mutants (Colot et al., 2006; Dunlap et al., 2007; McCluskey, 2003) that carried deletions in genes whose expression was affected (either increased or decreased) by exposure to PHS. Germinating conidia from most of them displayed no altered sensitivity to PHS. It is possible that the missing proteins are involved in PCD, but their role is redundant and can be complemented by other cellular proteins. However, we identified two deletion strains that displayed increased resistance to PHS compared with the wild-type strain, indicating that the absent proteins are involved in PCD. One of the deleted genes encodes a probable aldehyde dehydrogenase. The gene deleted in the second strain codes for a subunit of mitochondrial ATP synthase, connected with the respiratory chain. The identification of increased PHS resistance in these mutants is in line with our findings that mutants containing deletion mutations in genes encoding subunits of respiratory chain complex I also display increased resistance to PHS (Castro et al., 2008). Thus, the analysis of differential gene expression should prove very useful for the identification of novel components/pathways involved in PCD and for the design of drugs acting in the modulation of the process.

\section{ACKNOWLEDGEMENTS}

We would like to thank Artur Falcão for help with the spot assays. This research was supported by research grants from FCT from Portugal, the POCTI program of QCA III (co-participated by FEDER) and by a sabbatical fellowship from Fundação LusoAmericana to A.V. and a National Institutes of Health RO1 grant (GM60468) and a P01 multi-institutional program project grant (GM068087; Core III: Transcriptional profiling) to N. L. G.

\section{REFERENCES}

Benjamini, Y. \& Hochberg, Y. (1995). Controlling the false discovery rate - a practical and powerful approach to multiple testing. $J R$ Stat Soc Ser B Methodol 57, 289-300.

Bertrand, H. \& Pittenger, T. H. (1972). Isolation and classification of extranuclear mutants of Neurospora crassa. Genetics 71, 521-533.

Bonawitz, N. D., Rodeheffer, M. S. \& Shadel, G. S. (2006). Defective mitochondrial gene expression results in reactive oxygen species- 
mediated inhibition of respiration and reduction of yeast life span. Mol Cell Biol 26, 4818-4829.

Castro, A., Lemos, C., Falcao, A., Glass, N. L. \& Videira, A. (2008). Increased resistance of complex I mutants to phytosphingosineinduced programmed cell death. J Biol Chem 283, 19314-19321.

Cheng, J., Park, T.-S., Chio, L.-C., Fischl, A. S. \& Ye, X. S. (2003). Induction of apoptosis by sphingoid long-chain bases in Aspergillus nidulans. Mol Cell Biol 23, 163-177.

Cheng, W. C., Leach, K. M. \& Hardwick, J. M. (2008). Mitochondrial death pathways in yeast and mammalian cells. Biochim Biophys Acta 1783, 1272-1279.

Colot, H. V., Park, G., Turner, G. E., Ringelberg, C., Crew, C. M., Litvinkova, L., Weiss, R. L., Borkovich, K. A. \& Dunlap, J. C. (2006). A high-throughput gene knockout procedure for Neurospora reveals functions for multiple transcription factors. Proc Natl Acad Sci U S A 103, 10352-10357.

Coppola, G., Choi, S. H., Santos, M. M., Miranda, C. J., Tentler, D. Wexler, E. M., Pandolfo, M. \& Geschwind, D. H. (2006). Gene expression profiling in frataxin deficient mice: microarray evidence for significant expression changes without detectable neurodegeneration. Neurobiol Dis 22, 302-311.

Davis, R. H. \& de Serres, F. J. (1970). Genetic and microbiological research techniques for Neurospora crassa. Methods Enzymol 17A, 79-143.

Davis, R. H. \& Perkins, D. D. (2002). Timeline: Neurospora: a model of model microbes. Nat Rev Genet 3, 397-403.

Descheneau, A. T., Cleary, I. A. \& Nargang, F. E. (2005). Genetic evidence for a regulatory pathway controlling alternative oxidase production in Neurospora crassa. Genetics 169, 123-135.

Duarte, M. \& Videira, A. (2009). Effects of mitochondrial complex III disruption in the respiratory chain of Neurospora crassa. Mol Microbiol 72, 246-258.

Dunlap, J. C., Borkovich, K. A., Henn, M. R., Turner, G. E., Sachs, M. S., Glass, N. L., McCluskey, K., Plamann, M., Galagan, J. E. \& other authors (2007). Enabling a community to dissect an organism: overview of the Neurospora functional genomics project. Adv Genet 57, 49-96.

Eisenberg, T., Büttner, S., Kroemer, G. \& Madeo, F. (2007). The mitochondrial pathway in yeast apoptosis. Apoptosis 12, 1011-1023.

Fedorova, N. D., Badger, J. H., Robson, G. D., Wortman, J. R. \& Nierman, W. C. (2005). Comparative analysis of programmed cell death pathways in filamentous fungi. BMC Genomics 6, 177-190.

Fleissner, A. \& Glass, N. L. (2007). SO, a protein involved in hyphal fusion in Neurospora crassa, localizes to septal plugs. Eukaryot Cell 6, 84-94.

Frishman, D., Albermann, K., Hani, J., Heumann, K., Metanomski, A., Zollner, A. \& Mewes, H. W. (2001). Functional and structural genomics using PEDANT. Bioinformatics 17, 44-57.

Fröhlich, K. U., Fussi, H. \& Ruckenstuhl, C. (2007). Yeast apoptosis from genes to pathways. Semin Cancer Biol 17, 112-121.

Galagan, J. E., Calvo, S. E., Borkovich, K. A., Selker, E. U., Read, N. D., Jaffe, D., FitzHugh, W., Ma, L. J., Smirnov, S. \& other authors (2003). The genome sequence of the filamentous fungus Neurospora crassa. Nature 422, 859-868.

Glass, N. L. \& Dementhon, K. (2006). Non-self recognition and programmed cell death in filamentous fungi. Curr Opin Microbiol $\mathbf{9}$, 553-558.

Green, D. R. \& Kroemer, G. (2004). The pathophysiology of mitochondrial cell death. Science 305, 626-629.

Hamann, A., Brust, D. \& Osiewacz, H. D. (2008). Apoptosis pathways in fungal growth, development and ageing. Trends Microbiol 16, 276-283.

Hatefi, Y. (1985). The mitochondrial electron transport and oxidative phosphorylation system. Annu Rev Biochem 54, 1015-1069.
Kasuga, T. \& Glass, N. L. (2008). Dissecting colony development of Neurospora crassa using mRNA profiling and comparative genomics approaches. Eukaryot Cell 7, 1549-1564.

Kasuga, T., Townsend, J. P., Tian, C., Gilbert, L. B., Mannhaupt, G., Taylor, J. W. \& Glass, N. L. (2005). Long-oligomer microarray profiling in Neurospora crassa reveals the transcriptional program underlying biochemical and physiological events of conidial germination. Nucleic Acids Res 33, 6469-6485.

Kimchi, A. (2007). Programmed cell death: from novel gene discovery to studies on network connectivity and emerging biomedical implications. Cytokine Growth Factor Rev 18, 435-440.

Laun, P., Ramachandran, L., Jarolim, S., Herker, E., Liang, P., Wang, J., Weinberger, M., Burhans, D. T., Suter, B. \& other authors (2005). A comparison of the aging and apoptotic transcriptome of Saccharomyces cerevisiae. FEMS Yeast Res 5, 1261-1272.

Lu, B. C. K. (2006). Programmed cell death in fungi. In Growth, Differentiation and Sexuality. The Mycota, vol. I, 2nd edn, pp 167-187. Edited by U. Kues \& R. Fischer. Berlin: Springer.

Lucau-Danila, A., Lelandais, G., Kozovska, Z., Tanty, V., Delaveau, T., Devaux, F. \& Jacq, C. (2005). Early expression of yeast genes affected by chemical stress. Mol Cell Biol 25, 1860-1868.

Madeo, F., Herker, E., Wissing, S., Jungwirth, H., Eisenberg, T. \& Frohlich, K.-U. (2004). Apoptosis in yeast. Curr Opin Microbiol 7, 655-660.

Marques, I., Duarte, M., Assunção, J., Ushakova, A. V. \& Videira, A. (2005). Composition of complex I from Neurospora crassa and disruption of two "accessory" subunits. Biochem Biophys Acta 1707, 211-220.

McCluskey, K. (2003). The Fungal Genetics Stock Center: from molds to molecules. Adv Appl Microbiol 52, 245-262.

Metzenberg, R. L. (2004). Bird Medium: an alternative to Vogel Medium. Fungal Genet Newsl 51, 19-20.

Pereira, C., Silva, R. D., Saraiva, L., Johansson, B., Sousa, M. J. \& Côrte-Real, M. (2008). Mitochondria-dependent apoptosis in yeast. Biochim Biophys Acta 1783, 1286-1302.

Robson, G. D. (2006). Programmed cell death in the aspergilli and other filamentous fungi. Med Mycol 44, S109-S114.

Ruepp, A., Zollner, A., Maier, D., Albermann, K., Hani, J., Mokrejs, M., Tetko, I., Güldener, U., Mannhaupt, G. \& other authors (2004). The FunCat, a functional annotation scheme for systematic classification of proteins from whole genomes. Nucleic Acids Res 32, 5539-5545.

Seo, J. \& Shneiderman, B. (2002). Interactively exploring hierarchical clustering results. IEEE Comput 35, 80-86.

Skulachev, V. P. (2006). Bioenergetic aspects of apoptosis, necrosis and mitoptosis. Apoptosis 11, 473-485.

Tian, C., Kasuga, T., Sachs, M. S. \& Glass, N. L. (2007). Transcriptional profiling of cross pathway control in Neurospora crassa and comparative analysis of the Gcn4 and $\mathrm{CPC} 1$ regulons. Eukaryot Cell 6, 1018-1029.

Townsend, J. P. (2003). Multifactorial experimental design and the transitivity of ratios with spotted DNA microarrays. BMC Genomics 4, 41.

Townsend, J. P. \& Hartl, D. L. (2002). Bayesian analysis of gene expression levels: statistical quantification of relative mRNA level across multiple strains or treatments. Genome Biol 3, RESEARCH0071.

van der Westhuizen, F. H., van den Heuvel, L. P., Smeets, R., Veltman, J. A., Pfundt, R., van Kessel, A. G., Ursing, B. M. \& Smeitink, J. A. (2003). Human mitochondrial complex I deficiency: investigating transcriptional responses by microarray. Neuropediatrics 34, 14-22.

Woodcock, J. (2006). Sphingosine and ceramide signalling in apoptosis. IUBMB Life 58, 462-466.

Edited by: S. D. Harris 